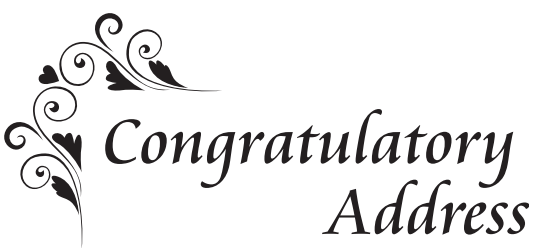

\title{
Fernando GARZON
}

On Behalf of The Board of Directors and the Members of The Electrochemical Society, I convey our sincere congratulations to the Electrochemical Society of Japan upon their 80th Anniversary. We salute the many achievements of the Society and its membership. The Electrochemical Society of Japan has been a leading contributor to the advancement of world science and technology throughout its distinguished history. As mankind faces the many challenges of increased population and energy usage, diminishing natural resources and the global environmental impacts associated with economic development, better technology needs to be implemented to ameliorate the undesirable consequences of our increasing standard of living. The Electrochemical Society of Japan, through its publications, technical symposia and the scientific breakthroughs of its membership, has played and will continue to play a vital role in advancing improvements in efficient energy conversion, energy storage, environmental sensing and monitoring, and green chemistry and manufacturing. These achievements are evident in our everyday lives. Among the many achievements are the advanced lithium battery technology in our mobile computers, telephones and tablets, the high efficiency and low emission hybrid vehicles we drive, emerging fuel cell energy conversion technology, the sensors that monitor our homes and workplaces for toxic and flammable gases, and the numerous new advanced medical devices that improve our health and vitality.

The Electrochemical Society and the Electrochemical Society of Japan have many mutual members and the societies have been formally collaborating for 40 years, this represents half of the historical lifetime of the Electrochemical Society of Japan. Our dual members were the basis of informal collaborations for decades before our joint meetings officially began. Our first international meeting was held October 1987 in Honolulu Hawaii after five years of planning between the leadership of our societies. The attendance has grown from 2500 to over 3800 participants at PRiME 2012 demonstrating the success and importance of our joint meetings. PRiME is the world's largest and most important meeting dedicated to electrochemical science and technology and we only expect it to grow in the future. International collaboration in scientific endeavors directed towards the benefit of humanity, As exemplified by the PRiME conference, will only become increasing more important for the future well being of the planet.

The Electrochemical Society would like to thank the outstanding past and present membership and leadership of the Electrochemical Society of Japan for their globally significant advancement of electrochemical science and technology and we wish you continued future success in your noble mission.

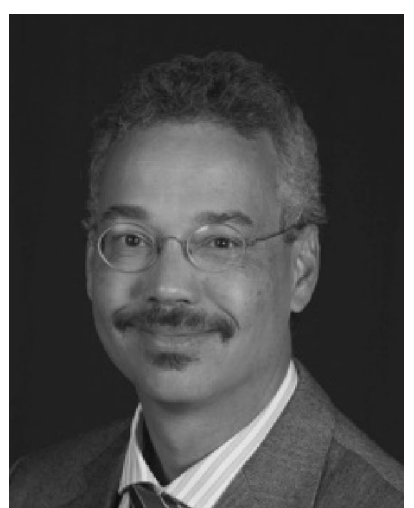

Fernando GARZON President of The Electrochemical Society Los Alamos National Laboratory 